\title{
Primary malignant hepatic paraganglioma mimicking liver tumor: A case report
}

\author{
ZHEN YOU, YILEI DENG, ANUJ SHRESTHA, FUYU LI and NANSHENG CHENG \\ Department of Biliary Surgery, West China Hospital, Sichuan University, Chengdu, Sichuan 610041, P.R. China
}

Received August 7, 2014; Accepted April 21, 2015

DOI: $10.3892 / 01.2015 .3318$

\begin{abstract}
An extra-adrenal pheochromocytoma is also known as a paraganglioma. The present study reported the case of a 47-year-old female patient with an extremely rare primary nonfunctioning hepatic paraganglioma without any clinical signs and symptoms. A computed tomography scan of the entire abdomen region revealed a hyper-enhanced, well-marginated round mass located in segment 3 of the liver. A preoperative diagnosis of hepatocellular carcinoma was established and a left lateral hepatic lobectomy was performed. During the removal of the mass, the patient experienced extreme fluctuations in blood pressure. Analysis of hepatic and peripheral venous blood test results confirmed the increase of noradrenaline secretion. Postoperatively, the patient's blood pressure and catecholamine level returned to the normal range. However, three years after surgery, a plasma catecholamine examination revealed a high noradrenaline level. Abdominal magnetic resonance imaging scans revealed two metastases, located in the spleen and below the right posterior lobe of the liver, which were identified as malignant paragangliomas. Therefore, the patient was diagnosed with primary malignant hepatic paraganglioma recurrence three years after hepatic resection.
\end{abstract}

\section{Introduction}

Paragangliomas, also known as extra-adrenal pheochromocytomas, are rare neuroendocrine tumors $(1,2)$. Patients may present with signs and symptoms of catecholamine excess, including paroxysmal hypertension, headache, palpitation and sweating, or may be completely asymptomatic (2-4). Although paragangliomas are usually benign tumors, arising from the paraganglionic tissue and distributed in areas between the neck and the pelvis, they have also been reported in other less common sites, including the gallbladder, lungs and cardiac area $(2,5-7)$. In general, complete surgical excisions are the

Correspondence to: Professor Nansheng Cheng, Department of Biliary Surgery, West China Hospital, Sichuan University, 37 Guoxue Road, Chengdu, Sichuan 610041, P.R. China

E-mail: nanshengcheng2000@yeah.net

Key words: paraganglioma, primary, liver conventional treatment for these tumors. However, for malignant paragangliomas, surgical debulking is rarely curative and is only considered as a palliative therapy $(2,3)$. In the present study, the case of an extremely rare primary nonfunctioning hepatic paraganglioma, mimicking a primary liver tumor, was described. The patient underwent partial hepatectomy. Although extremely rare cases of primary hepatic paranglioma have been described previously (7-11), knowledge of the present case is valuable in order to help avoid the possibility of confusing hepatic paraganglioma with other liver tumors.

\section{Case report}

The study was approved by the Ethics Committee of West China Hospital, and written informed consent was obtained from the patient. In September 2010, an asymptomatic 47-year-old female patient was admitted to the West China Hospital (Chengdu, China) with an abdominal ultrasound (US) diagnosis of a solitary left hepatic lesion. The patient had no history of drug or alcohol abuse, previous malignancies or weight loss. Physical examination revealed no abnormal findings, with the exception of mild hypertension $(150 / 100 \mathrm{mmHg})$. The patient's blood pressure was reduced to $\sim 140 / 90 \mathrm{mmHg}$ by administration of felodipine (10 mg/day). Laboratory values, including serum bilirubin and transaminases (aspartate transaminase, alanine transaminase), blood urea, nitrogen and serum creatinine were all within the normal range, indicating normal liver and renal function; hepatitis serology and $\alpha$-fetoprotein levels were also within the normal range. A contrast-enhanced helical computed tomography (CT) scan of the entire abdomen revealed a hypodense, well-marginated round mass that measured $3.6 \times 3.4 \mathrm{~cm}$ and was located in segment 3 of the liver in a subcapsular location. The mass was highly vascular and markedly enhanced following intravenous injection of iodine (Fig. 1A and B), and a preoperative diagnosis of hepatocellular carcinoma was established. No abnormalities were noted in the kidneys and adrenal glands. Subsequently, a left lateral hepatic lobectomy was performed. Intraoperatively, a dark red, multinodular solid mass that measured $\sim 4.0 \mathrm{~cm}$ in diameter was observed. A thorough exploration of the abdominal cavity did not reveal any other abnormalities. During the removal of the mass, the patient experienced extreme fluctuations in blood pressure in the range of 90/70-310/200 $\mathrm{mmHg}$. Therefore, the endocrine activity of the mass was suspected. Analysis of hepatic and peripheral venous blood samples confirmed the 
A

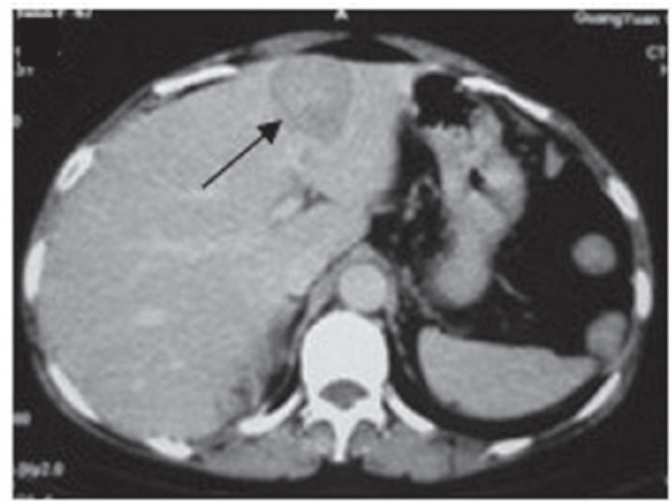

C

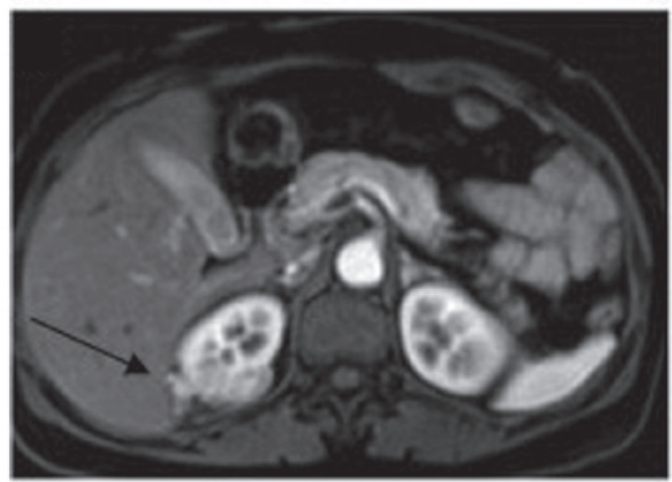

B

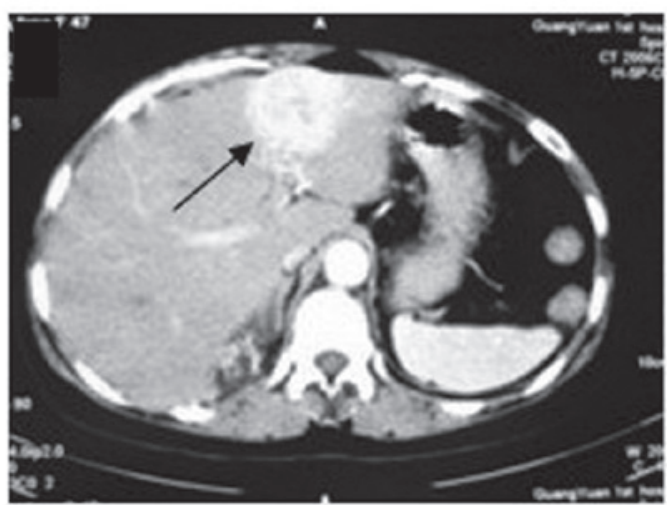

D

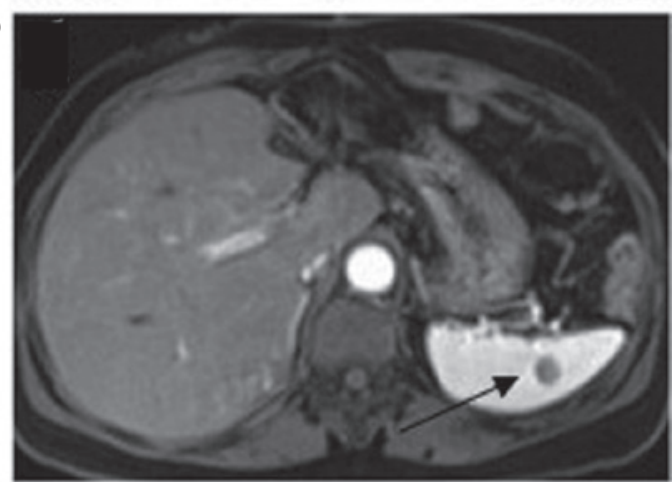

Figure 1. Preoperative CT scans of the primary malignant hepatic paraganglioma: (A) Non-enhanced CT, and (B) Enhanced CT scanning, revealed a hypodense, well-marginated round mass, measuring 3.6x3.4 cm. The mass was located in segment 3 of the liver in a subcapsular location and was markedly enhanced following intravenous injection of iodine (arrow). Abdominal MRI scans three years after the left lateral hepatic lobectomy for hepatic paraganglioma revealed two metastases: (C) One lesion with unclear boundaries below the liver right-posterior lobe, which demonstrated a high-intensity signal on T1-weighted image (arrow); and (D) a second mass located in the spleen with slight high-intensity signal on the T1-weighted image (arrow). CT, computed tomography; MRI, magnetic resonance imaging.

increase of noradrenaline secretion $[1627 \mathrm{ng} / \mathrm{l}$ and $1312 \mathrm{ng} / \mathrm{l}$, respectively; normal range, 174-357 ng/l (12)]. Postoperatively, the patient's blood pressure gradually stabilized to $130 / 76 \mathrm{mmHg}$ without administration of any antihypertensive drugs, and the concentration of catecholamine in the peripheral blood was reduced to the normal range 5 days after surgery. Immunohistochemical analysis of the resected tissue revealed that the mass was positive for chromogranin A and neuron-specific enolase. Sustentacular cells that were positive for S100 protein were also identified. However, the tissues were negative for synaptophysin, cytokeratin-8, epithelial membrane antigen, HMB45, MART1 and Hep Par 1 values. The overall immunohistochemical findings supported the diagnosis of paraganglioma.

Within the initial two years of follow-up, the blood pressure of the patient and the results of repeated plasma catecholamine tests were found to be within the normal range. Abdominal US was performed every three months and demonstrated no evidence of recurrence or metastasis. Thereafter, regular telephone follow-ups also revealed that the patient was in the good general condition. On April 2014, the patient attended an outpatient follow-up visit, during which the plasma catecholamine examination revealed a high noradrenaline level of $2286 \mathrm{ng} / \mathrm{ml}$. However, the patient was asymptomatic and the blood pressure was stable at $140 / 78 \mathrm{mmHg}$. Subsequently, abdominal magnetic resonance imaging (MRI) scans were performed, which revealed two metastases (Fig. 1C and D). One lesion with unclear boundaries was identified below the right posterior lobe of the liver and exhibited a high-intensity signal on T1- and T2- weighted images. The other lesion was located in the spleen, exhibiting a slight high-intensity signal on the T1-weighted image and equal signal intensity to the spleen on the T2-weighted image. The metastases were established to be malignant paragangliomas (3). Therefore, the patient was diagnosed with primary malignant hepatic paraganglioma recurrence three years after hepatic resection. Subsequently, appropriate radiotherapy were recommended for this patient, but she refused further treatment and also refused additional follow-up without definite cause.

\section{Discussion}

Primary hepatic paraganglioma is an extremely rare tumor. The majority of hepatic paragangliomas are metastatic, since the liver is the second most frequent site of metastasis from malignant pheochromocytoma (13). In the present study, the patient was diagnosed with hepatic paraganglioma, based on the following three main arguments: i) Absence of any other tumor in the chest, and abdominal or pelvic cavities; ii) increase of noradrenaline secretion in the hepatic and peripheral venous blood, followed by reduction to the normal levels at least two years after the primary tumor resection; and iii) a follow-up period without any evidence of recurrence, with tumor recurrence detected three years after surgery. A radiologic diagnosis of hepatic pheochromocytoma based on CT or MRI scans is not normally possible, due to the nonspecific 
radiologic features of this tumor (7). Metaiodobenzylguanidine (MIBG) scanning has been previously demonstrated to have a high specificity and sensitivity for detecting pheochromocytoma (14). However, in the present case, hepatic paraganglioma was not suspected preoperatively, since the radiologic features (in CT scans) of this tumor were nonspecific and the patient did not present any characteristic symptoms of catecholamine excess, including marked hypertension, headache or palpitation (2-4). In patients with liver masses, particularly those with unexplained hypertension, MIBG is a valuable test with high specificity for detecting a paraganglioma, when this tumor is suspected (14).

The majority of abdominal paragangliomas are benign; however, at least $15-35 \%$ of cases are malignant $(15,16)$. The clinical, biochemical and radiological features are not adequate to predict malignancy or distinguish benign from malignant lesions $(2,17)$. However, the presence of distant metastases, such as in the liver, lungs and/or bones, indicates malignancy. Metastases may be identified at the time of the initial diagnosis and surgery, or may only become evident following surgical removal of the primary tumor, typically within five years (17). In the current case, the metastases were not present until three years after surgery. Therefore, the follow-up period should be long in order to detect such metastases. At present, there is no universally effective therapeutic strategy for malignant paragangliomas. Surgical resection of a primary mass or metastases is rarely curative, but it may be effective in obtaining rapid palliation of the symptoms. Furthermore, surgical debulking may be used prior to radio- or chemotherapy; however, whether this strategy increases survival requires further clinical assessment in randomized prospective trials $(2,17)$.

The current case study reported an extremely rare case of primary malignant hepatic paraganglioma without typical signs and symptoms of catecholamine excess that are normally expected when blood levels of catecholamine are elevated. The paraganglioma was successfully resected, and the levels of circulating catecholamine were reduced to within the normal range and the blood pressure was stable following surgery, but recurred 3 years following the resection. The present case indicates that despite the rarity, hepatic paraganglioma should be considered in the differential diagnosis of liver tumor, particularly when the blood levels of catecholamine and/or the blood pressure is elevated. Surgery for malignant hepatic pheochromocytoma is rarely curative; however, it may quickly reduce the levels of circulating catecholamine and offer palliation of symptoms.

\section{Acknowledgements}

The authors would like to acknowledge the support from the Department of Pathology at the West China Hospital. This work was supported by the Science \& Technology Support Project of Sichuan Province (No. 2011FZ2009).

\section{References}

1. Whalen RK, Althausen AF and Daniels GH: Extra-adrenal pheochromocytoma. J Urol 147: 1-10, 1992.

2. Young WF Jr: Paragangliomas: Clinical overview. Ann N Y Acad Sci 1073: 21-29, 2006.

3. Chrisoulidou A, Kaltsas G, Ilias I and Grossman AB: The diagnosis and management of malignant phaeochromocytoma and paraganglioma. Endocr Relat Cancer 14: 569-585, 2007.

4. Ahmad S1, Cathy D, Sheikh M and Sweeney P: Retroperitoneal extra-adrenal paraganglioma: a rare but important diagnosis. Ir J Med Sci 178: 211-214, 2009.

5. Freschi M1 and Sassi I: Paraganglioma of the gallbladder. Pathologica 82: 459-463, 1990 (Article in Italian).

6. Jurascheck F, Egloff H, Buemi A and Laedlein-Greilsammer D: Paraganglioma of urinary bladder. Urology 22: 659-663, 1983.

7. Roman SA and Sosa JA: Functional paragangliomas presenting as primary liver tumors. South Med J 100: 195-196, 2007.

8. Chang H, Xu L and Mu Q: Primary functioning hepatic paraganglioma: A case report. Adv Ther 23: 817-820, 2006.

9. Corti B, D'Errico A, Pierangeli F, Fiorentino M, Altimari A, and Grigioni WF: Primary paraganglioma strictly confined to the liver and mimicking hepatocellular carcinoma: an immunohistochemical and in situ hybridization study. Am J Surg Pathol 26: 945-949, 2002.

10. Reif MC1, Hanto DW, Moulton JS, Alspaugh JP and Bejarano P: Primary hepatic pheochromocytoma? Am J Hypertens 9: 10401043, 1996.

11. Kang YS, Kuhlman JE, Fishman EK and Zerhouni EA: Nonfunctional paraganglioma of the liver. Clin Imaging 15: 216-219, 1991.

12. Eisenhofer G1, Goldstein DS, Kopin IJ and Crout JR: Pheochromocytoma: rediscovery as a catecholamine-metabolizing tumor. Endocr Pathol 14: 193-212, 2003.

13. Schlumberger M, Gicquel C, Lumbroso J, et al: Malignant pheochromocytoma: Clinical, biological, histologic and therapeutic data in a series of 20 patients with distant metastases. $J$ Endocrinol Invest 15: 631-642, 1992.

14. Ilias I and Pacak K: Anatomical and functional imaging of metastatic pheochromocytoma. Ann N Y Acad Sci 1018: 495-504, 2004.

15. O'Riordain DS1, Young WF Jr, Grant CS, Carney JA and van Heerden JA: Clinical spectrum and outcome of functional extraadrenal paraganglioma. World J Surg 20: 916-922, 1996.

16. Chrisoulidou A1, Kaltsas G, Ilias I and Grossman AB: The diagnosis and management of malignant phaeochromocytoma and paraganglioma. Endocr Relat Cancer 14, 569-585, 2007.

17. Eisenhofer G, Bornstein SR, Brouwers FM, et al: Malignant pheochromocytoma: Current status and initiatives for future progress. Endocr Relat Cancer 11: 423-436, 2004. 